\title{
Phosphocalyculin C as a Pyrophosphate Protoxin of Calyculin C in the Marine Sponge Discodermia calyx
}

\author{
Yoko Egami, Toshiyuki Wakimoto, ${ }^{*}$ Ikuro Abe
}

Graduate School of Pharmaceutical Sciences, The University of Tokyo, 7-3-1 Hongo, Bunkyo-ku, Tokyo 113-0033, Japan

wakimoto@mol.f.u-tokyo.ac.jp, abei@mol.f.u-tokyo.ac.jp

\section{RECEIVED DATE}

TITLE RUNNING HEAD: Isolation of phosphocalyculin C

*Address correspondence to this author. Phone: +81-3-5841-4740. Fax: +81-3-5841-4744.

ABSTRACT: Calyculin C, a minor derivative of the calyculins, has an additional methyl group on C32 of calyculin A. A recent biosynthetic study of calyculins revealed that an end product of calyculin biosynthesis is the pyrophosphate form, phosphocalyculin A. However, the pyrophosphate counterpart derived from calyculin $\mathrm{C}$ had not been reported. We isolated phosphocalyculin $\mathrm{C}$ as a minor pyrophosphate derivative, by a detailed investigation of an extract from the sponge Discodermia calyx. The treatment of phosphocalyculin C with the D. calyx cell-free extract significantly enhanced its cytotoxicity, providing molecular evidence for its role as the protoxin of calyculin $\mathrm{C}$.

KEYWORDS: Phosphocalyculin C, Calyculin C, Discodermia calyx, Prodrug 
Calyculin A (1, Fig. 1), the major constituent of the Japanese marine sponge Discodermia calyx, is a potent cytotoxin that was shown to be a specific inhibitor of protein phosphatases 1 and $2 \mathrm{~A}$. $^{1-5}$ In terms of the tetraene moiety of $\mathbf{1}$, a series of geometrical isomers, calyculins $\mathrm{B}$, $\mathrm{E}$, and $\mathrm{F}$, are photochemically derived from calyculin A. ${ }^{6,7}$ In addition to these isomers, another minor series of geometrical isomers, calyculins $\mathrm{D}, \mathrm{G}$, and $\mathrm{H}$, are also known, and they are all generated by the photoisomerization of calyculin C (2, Fig. 1), which has an additional methyl group on C32 of calyculin A. Thus, $\mathbf{1}$ and $\mathbf{2}$ seemed to be the parents of two series of geometrical isomers in the sponge extract. Recently, we reported the calyculin biosynthetic gene cluster, identified by a metagenomic mining approach, and the sponge symbiont, Candidatus "Entotheonella sp.", as the producer of calyculins. ${ }^{8}$ The C32-33 moiety of calyculins is likely to be constructed by a nonribosomal peptide synthetase (NRPS) module, in which the adenylation (A) domain exhibits a similar NRPS code to the Gly consensus sequence, but four of the ten residues are different. Therefore, we anticipated that the promiscuity of this A domain renders it able to accept not only Gly but also Ala, to a lesser extent, to generate the corresponding moieties of $\mathbf{1}$ and 2, respectively. In addition to the genetic background of calyculin biosynthesis, we revealed that the end product of calyculin biosynthesis was the pyrophosphate form, rather than the monophosphate such as $\mathbf{1}$, by the functional analysis of a phosphotransferase, CalQ, encoded in the calyculin biosynthetic gene cluster, as well as the reinvestigation of the sponge extract. These findings suggested that the pyrophosphate form of 2 also exists as the intact form in the sponge Discodermia calyx. Herein, we report the isolation, structure elucidation and biological activity of phosphocalyculin C (Fig. 1).

The inherent phosphatase activity in the sponge tissue, which quickly liberates the phosphoryl group of pyrophosphate during the extraction step, prevents the isolation of phosphocalyculins, and instead, only the monophosphate forms, such as $\mathbf{1}$ and 2, had been detected in the conventional extraction procedure. On the other hand, the flash-freezing of the sponge specimen prior to extraction was required to detect phosphocalyculin A (3, Fig. 1) as the dominant metabolite, since this strategy avoids the tissue disruption inducing the endogenous phosphatase activity. As shown in Figure 2, the 
metabolic profiles between the frozen tissue and the lyophilized tissue after flash-freezing in liquid nitrogen were significantly different, on the basis of the comparative analysis by ODS-HPLC. Although $\mathbf{1}$ and $\mathbf{2}$ were detected as the major and minor metabolites in the frozen tissue extracts, respectively (Fig. 2a), an uncharacterized peak (4) in addition to 3 was detected in the lyophilized tissue (Fig. 2b). The LC-MS analysis revealed that the pseudomolecular ion of this unknown compound was 80 mass units higher than that observed for 2 . Since the difference in the mass units implied a phosphate group, we predicted it would be phosphocalyculin C (4). To isolate this compound, freshly obtained D. calyx sponges were flash frozen in liquid nitrogen, immediately lyophilized ( $80 \mathrm{~g}$, dry weight), and then extracted with methanol. The methanol extract was directly fractionated by gel-filtration column chromatography (Sephadex LH-20; $2.5 \times 75 \mathrm{~cm}$ ) with methanol. The fractions containing 3 and 4 were purified by RP HPLC (Cosmosil MS-II; 10 x $250 \mathrm{~mm}$ ), with $45 \% \mathrm{CH}_{3} \mathrm{CN}$ containing $0.1 \%$ TFA, followed by $80 \% \mathrm{CH}_{3} \mathrm{OH}$ containing $0.1 \%$ TFA, to yield $4(6.2 \mathrm{mg}) ;[\alpha]_{\mathrm{D}}^{23}=-49\left(c=0.25\right.$ in $\left.\mathrm{CH}_{3} \mathrm{OH}\right)$; HRFABMS (m/z): $[\mathrm{M}+\mathrm{Na}]^{+}$calcd for $\mathrm{C}_{51} \mathrm{H}_{85} \mathrm{~N}_{4} \mathrm{O}_{18} \mathrm{P}_{2}, 1125.5154$; found, 1125.5145 .

The ${ }^{1} \mathrm{H}$ NMR spectrum of $\mathbf{4}$ displayed five doublet methyl signals (Table 1, Fig. S1), which indicated the presence of an additional methyl group on C32. The ${ }^{13} \mathrm{C}$ NMR spectrum exhibited two doublet signals corresponding to $\mathrm{C} 17$ and $\mathrm{C} 16$, but all other oxygenated carbon signals were singlets (Fig. S2). Both spectra were superimposable with those of 2, except for the proton and carbon signals for the positions around the oxazole moiety (Table 1), as observed for $\mathbf{1}$ and $\mathbf{3}$. The proton-decoupled ${ }^{31} \mathrm{P}$ NMR spectrum displayed two doublet signals at $\delta_{\mathrm{P}}-10.5$ and -10.7 (Fig. S3), which are diagnostic for a pyrophosphate functionality. To confirm the stereochemistry of $\mathbf{4}$, we then performed the enzymatic transformation of $\mathbf{2}$ to $\mathbf{4}$, by using the phosphotransferase CalQ, which was previously reported to catalyze the phosphorylation of 1 to produce $3 .{ }^{8}$ As a result, HPLC and LC-MS analyses revealed that $\mathbf{4}$ was identical to the pyrophosphate form of $\mathbf{2}$, generated by the CalQ-catalyzed reaction (Fig. 2c), thus corroborating the stereochemical coincidence between $\mathbf{2}$ and $\mathbf{4}$.

Cytotoxicity assays against $\mathrm{P} 388$ murine leukemia cells revealed that $\mathbf{4}$, with an $\mathrm{IC}_{50}$ value of 36 $\mathrm{nM}$, was at least 5,000-fold less cytotoxic than $2\left(\mathrm{IC}_{50} ; 7 \mathrm{pM}\right)$ (Fig. 3). These results suggested that the 
less active phosphocalyculin C plays the role of a protoxin, as in the cases of precolibactin ${ }^{9}$ or the didemnins $\mathrm{X}$ and $\mathrm{Y}^{10}$, which were isolated as NRPS- and/or PKS-derived natural prodrugs of the cytotoxins colibactin and didemin $\mathrm{B}$, respectively. To demonstrate the protoxin activation process, 4 was treated with a D. calyx cell-free extract prior to the treatment of tumor cells. As expected, the pretreatment of 4 with $100 \mathrm{ng} / \mathrm{mL}$ of $D$. calyx cell-free extract significantly enhanced the cytotoxicity, with a 1,000 -fold lower $\mathrm{IC}_{50}$ value than that of 4 itself, while the $D$. calyx cell-free preparation, lacking small molecules such as calyculins, exhibited no cytotoxicity even at the concentration of $1 \mathrm{mg} / \mathrm{mL}$. In previous studies, we revealed that an as-yet-unidentified phosphatase, catalyzing the bioconversion of $\mathbf{3}$ into 1 in response to tissue wounding, exists in $D$. calyx $^{8}$ Taken together, the enhanced cytotoxicity of 4 by the treatment with the cell-free extract is considered to result from the generation of the mature toxin 2, by dephosphorylation. More detailed investigations of the activation mechanism of the calyculin prodrug may provide significant opportunities for the development of cancer therapies by the on-site exposure of cytotoxins targeting cells, in combination with a gene-directed enzyme prodrug therapy (GDEPT) or an antibody-directed enzyme prodrug therapy (ADEPT). ${ }^{11}$

ACKNOWLEDGMENTS This work was partly supported by the Nagase Science Technology Foundation, the Astellas Foundation for Research on Metabolic Disorders, the CREST program from Japan Science and Technology Agency, the Bilateral Program between Japan and Switzerland from the Japan Society for the Promotion of Science (JSPS), and Grants-in-Aid from the Ministry of Education, Culture, Sports, Science and Technology (MEXT), Japan. 


\section{REFERENCES}

(1) Kato, Y.; Fusetani, N.; Matsunaga, S.; Hashimoto, K.; Fujita, S.; Furuya, T. J. Am. Chem. Soc. 1986, 108,2780 .

(2) Döderlein, L. H. P. Z. Wiss. Zool. Abt. A. 1884, 40, 62.

(3) Ishihara, H.; Martin, B. L.; Brautigan, D. L.; Karaki. H.; Ozaki, H.; Kato, Y.; Fusetani, N.; Watabe, S.; Hashimoto, K.; Uemura, D.; Hartshorne, D. J. Biochem. Biophys. Res. Commun. 1989, 159, 871 .

(4) Wakimoto, T.; Matsunaga, S.; Takai, A.; Fusetani, N. Chem. Biol. 2002, 9, 309.

(5) Kita, A.; Matsunaga, S.; Takai, A.; Kataiwa, H.; Wakimoto, T.; Fusetani, N.; Isobe, M.; Miki, K. Structure 2002, 10, 715.

(6) Kato, Y.; Fusetani, N.; Matsunaga, S.; Hashimoto, K.; Koseki, K. J. Org. Chem. 1988, 53, 3930.

(7) Matsunaga, S.; Fujiki, H.; Sakata, D.; Fusetani, N. Tetrahedron 1991, 47, 2999.

(8) Wakimoto, T.; Egami, Y.; Nakashima, Y.; Wakimoto, Y.; Mori, T.; Awakawa,T.; Ito, T.; Kenmoku, H.; Asakawa, Y.; Piel, J.; Abe, I. $\quad$ Nat. Chem. Biol. 2014, 10, 648.

(9) Brotherton, C. A.; Balskus, E. P. J. Am. Chem. Soc. 2013, 135, 3359.

(10) Xu, Y.; Kersten, R. D.; Nam, S. J.; Lu, L.; Al-Suwailem, A. M.; Zheng, H.; Fenical, W.; Dorrestein, P. C.; Moore B. S.; Qian, P. Y. J. Am. Chem. Soc. 2012, 134, 8625.

(11) Tietze, L. F.; Schmuck, K. Curr. Pharm. Des. 2011, 17, 3527. 


\begin{tabular}{|c|c|c|c|c|c|c|}
\hline \multirow{2}{*}{ Position } & \multicolumn{2}{|c|}{${ }^{13} \mathrm{C}(\mathrm{J} \mathrm{Hz})$} & \multicolumn{2}{|c|}{${ }^{1} \mathrm{H}(\mathrm{J} \mathrm{Hz})$} & \multicolumn{2}{|c|}{${ }^{31} \mathrm{P}(J \mathrm{~Hz})$} \\
\hline & 4 & 2 & 4 & 2 & 4 & 2 \\
\hline 1 & 117.0 & 116.9 & - & - & - & - \\
\hline 2 & 94.1 & 94.1 & $5.27(1 \mathrm{H}, \mathrm{s})$ & $5.27(1 \mathrm{H}, \mathrm{s})$ & - & - \\
\hline 3 & 157.4 & 157.3 & - & - & - & - \\
\hline 4 & 128.2 & 128.1 & $6.75(1 \mathrm{H}, \mathrm{d}, 15.3)$ & $6.75(1 \mathrm{H}, \mathrm{d}, 14.7)$ & - & - \\
\hline 5 & 133.9 & 134.6 & $7.12(1 \mathrm{H}, \mathrm{dd}, 10.8,15.3)$ & $7.13(1 \mathrm{H}, \mathrm{dd}, 11.3,14.7)$ & - & - \\
\hline 6 & 124.1 & 123.8 & $6.34(1 \mathrm{H}, \mathrm{d}, 11.3)$ & $6.36(1 \mathrm{H}, \mathrm{d}, 10.8)$ & - & - \\
\hline 7 & 144.5 & 144.6 & - & - & - & - \\
\hline 8 & 135.5 & 133.9 & - & - & - & - \\
\hline 9 & 131.0 & 131.4 & $6.02(1 \mathrm{H}, \mathrm{d}, 9.6)$ & $6.08(1 \mathrm{H}, \mathrm{d}, 9.6)$ & - & - \\
\hline 10 & 35.8 & 33.6 & $2.73(1 \mathrm{H}, \mathrm{ddq}, 2.3,9.6,7.4)$ & $2.77(1 \mathrm{H}, \mathrm{ddq}, 2.3,9.6,6.8)$ & - & - \\
\hline 11 & 77.5 & 79.7 & $3.35(1 \mathrm{H}, \mathrm{dd}, 2.3,9.6)$ & $3.44(1 \mathrm{H}, \mathrm{dd}, 2.3,9.6)$ & - & - \\
\hline 12 & 43.6 & 42.3 & $1.56(1 \mathrm{H}, \mathrm{m})$ & $1.58(1 \mathrm{H}, \mathrm{m})$ & - & - \\
\hline 13 & 70.2 & 73.2 & $3.73(1 \mathrm{H}, \mathrm{m})$ & $3.73(1 \mathrm{H}, \mathrm{m})$ & - & - \\
\hline $14 \mathrm{a}$ & 35.0 & 36.4 & $1.39(1 \mathrm{H}, \mathrm{m})$ & $1.53(1 \mathrm{H}, \mathrm{m})$ & - & - \\
\hline $14 \mathrm{~b}$ & - & - & $1.52(1 \mathrm{H}, \mathrm{m})$ & $1.77(1 \mathrm{H}, \mathrm{m})$ & - & - \\
\hline 15 & 78.7 & 78.0 & $3.64(1 \mathrm{H}, \mathrm{m})$ & $3.80(1 \mathrm{H}, \mathrm{m})$ & - & - \\
\hline 16 & $84.4(\mathrm{~d}, 6.0)^{a}$ & $84.2(\mathrm{~d}, 6.0)^{a}$ & $4.07\left(1 \mathrm{H}, \mathrm{ddd}, 1.9^{c}, 4.8,9.1\right)$ & $4.08(1 \mathrm{H}, \mathrm{ddd}, 1.9,4.0,9.1)$ & - & - \\
\hline 17 & $84.6(\mathrm{~d}, 6.0)^{b}$ & $82.5(\mathrm{~d}, 6.0)^{b}$ & $4.52\left(1 \mathrm{H}, \mathrm{dd}, 4.8,10.2^{d}\right)$ & $4.29\left(1 \mathrm{H}, \mathrm{dd}, 4.0,10.2^{d}\right)$ & - & - \\
\hline 18 & 50.4 & 50.3 & - & - & - & - \\
\hline 19 & 108.5 & 108.5 & - & - & - & - \\
\hline $20 \mathrm{a}$ & 29.4 & 29.4 & $1.59(1 \mathrm{H}, \mathrm{m})$ & $1.60(1 \mathrm{H}, \mathrm{m})$ & - & - \\
\hline $20 \mathrm{~b}$ & - & - & $1.88(1 \mathrm{H}, \mathrm{m})$ & $1.89(1 \mathrm{H}, \mathrm{m})$ & - & - \\
\hline 21 & 70.9 & 71.2 & $3.90(1 \mathrm{H}, \mathrm{m})$ & $3.85(1 \mathrm{H}, \mathrm{m})$ & - & - \\
\hline 22 & 37.7 & 37.5 & $1.64(1 \mathrm{H}, \mathrm{m})$ & $1.61(1 \mathrm{H}, \mathrm{m})$ & - & - \\
\hline 23 & 67.8 & 67.1 & $4.42(1 \mathrm{H}, \mathrm{dt}, 11.9,2.3)$ & $4.38(1 \mathrm{H}, \mathrm{dt}, 11.9,2.3)$ & - & - \\
\hline $24 \mathrm{a}$ & 35.9 & 35.8 & $2.05(1 \mathrm{H}, \mathrm{m})$ & $2.05(1 \mathrm{H}, \mathrm{m})$ & - & - \\
\hline $24 \mathrm{~b}$ & - & - & $2.52(1 \mathrm{H}, \mathrm{m})$ & $2.44(1 \mathrm{H}, \mathrm{m})$ & - & - \\
\hline 25 & 137.1 & 132.3 & $7.05(1 \mathrm{H}, \mathrm{ddd}, 4.5,10.8,16.2)$ & $6.82(1 \mathrm{H}, \mathrm{ddd}, 4.5,11.3,17.0)$ & - & - \\
\hline 26 & 113.6 & 116.6 & $6.28(1 \mathrm{H}, \mathrm{d}, 16.2)$ & $6.24(1 \mathrm{H}, \mathrm{d}, 17.0)$ & - & - \\
\hline 27 & 133.6 & 137.6 & - & - & - & - \\
\hline 28 & 135.9 & 135.3 & $7.93(1 \mathrm{H}, \mathrm{s})$ & $7.68(1 \mathrm{H}, \mathrm{s})$ & - & - \\
\hline 29 & 171.0 & 169.7 & - & - & - & - \\
\hline 30 & 29.1 & 29.8 & $3.60(1 \mathrm{H}, \mathrm{m})$ & $3.21(1 \mathrm{H}, \mathrm{m})$ & - & - \\
\hline $31 \mathrm{a}$ & 38.2 & 37.9 & $1.73(1 \mathrm{H}, \mathrm{m})$ & $1.77(1 \mathrm{H}, \mathrm{m})$ & - & - \\
\hline $31 \mathrm{~b}$ & - & - & $2.22(1 \mathrm{H}, \mathrm{m})$ & $1.92(1 \mathrm{H}, \mathrm{m})$ & - & - \\
\hline 32 & 41.9 & 41.5 & $4.14(1 \mathrm{H}, \mathrm{m})$ & $4.22(1 \mathrm{H}, \mathrm{m})$ & - & - \\
\hline $32 \mathrm{Me}$ & 20.6 & 20.1 & $1.12(3 \mathrm{H}, \mathrm{d}, 6.8)$ & $1.23(3 \mathrm{H}, \mathrm{d}, 6.2)$ & - & - \\
\hline 33 & 174.3 & 174.9 & - & - & - & - \\
\hline 34 & 72.0 & 70.2 & $4.00(1 \mathrm{H}, \mathrm{d}, 8.8)$ & $3.84(1 \mathrm{H}, \mathrm{d}, 9.1)$ & - & - \\
\hline 35 & 69.9 & 71.9 & $4.46(1 \mathrm{H}, \mathrm{dd}, 2.3,8.8)$ & $4.39(1 \mathrm{H}, \mathrm{dd}, 2.3,9.1)$ & - & - \\
\hline 36 & 65.7 & 65.0 & $3.93(1 \mathrm{H}, \mathrm{m})$ & $3.85(1 \mathrm{H}, \mathrm{m})$ & - & - \\
\hline $37 \mathrm{a}$ & 65.8 & 64.7 & $3.96(1 \mathrm{H}, \mathrm{m})$ & $3.92(1 \mathrm{H}, \mathrm{m})$ & - & - \\
\hline $37 \mathrm{~b}$ & - & - & $3.97(1 \mathrm{H}, \mathrm{m})$ & $3.94(1 \mathrm{H}, \mathrm{m})$ & - & - \\
\hline 38 & 58.0 & 58.0 & $3.42(3 \mathrm{H}, \mathrm{s})$ & $3.42(3 \mathrm{H}, \mathrm{s})$ & - & - \\
\hline 39 & 39.2 & 39.6 & $2.96(3 \mathrm{H}, \mathrm{s})$ & $2.93(3 \mathrm{H}, \mathrm{s})$ & - & - \\
\hline 40 & 43.2 & 42.9 & $2.90(3 \mathrm{H}, \mathrm{s})$ & $2.91(3 \mathrm{H}, \mathrm{s})$ & - & - \\
\hline 41 & 16.4 & 17.2 & $1.33(3 \mathrm{H}, \mathrm{d}, 6.8)$ & $1.30(3 \mathrm{H}, \mathrm{d}, 7.4)$ & - & - \\
\hline 42 & 9.6 & 9.7 & $0.91(3 \mathrm{H}, \mathrm{d}, 6.8)$ & $0.90(3 \mathrm{H}, \mathrm{d}, 7.4)$ & - & - \\
\hline 43 & 21.8 & 21.4 & $0.96(3 \mathrm{H}, \mathrm{s})$ & $0.97(3 \mathrm{H}, \mathrm{s})$ & - & - \\
\hline 44 & 16.8 & 16.8 & $1.26(3 \mathrm{H}, \mathrm{s})$ & $1.23(3 \mathrm{H}, \mathrm{s})$ & - & - \\
\hline 45 & 59.7 & 59.8 & $3.53(3 \mathrm{H}, \mathrm{s})$ & $3.52(3 \mathrm{H}, \mathrm{s})$ & - & - \\
\hline 46 & 10.4 & 11.1 & $0.72(3 \mathrm{H}, \mathrm{d}, 7.4)$ & $0.68(3 \mathrm{H}, \mathrm{d}, 6.8)$ & - & - \\
\hline 47 & 17.3 & 17.4 & $1.03(3 \mathrm{H}, \mathrm{d}, 7.4)$ & $1.04(3 \mathrm{H}, \mathrm{d}, 6.8)$ & - & - \\
\hline 48 & 13.0 & 12.1 & $1.85(3 \mathrm{H}, \mathrm{s})$ & $1.87(3 \mathrm{H}, \mathrm{s})$ & - & - \\
\hline 49 & 13.7 & 13.6 & $2.02(3 \mathrm{H}, \mathrm{s})$ & $2.03(3 \mathrm{H}, \mathrm{s})$ & - & - \\
\hline 50 & 18.1 & 18.0 & $2.09(3 \mathrm{H}, \mathrm{s})$ & $2.08(3 \mathrm{H}, \mathrm{s})$ & - & - \\
\hline P1 & - & - & - & - & $-10.47(\mathrm{~d}, 16.1)^{e}$ & $-0.62(\mathrm{~s})$ \\
\hline $\mathrm{P} 2$ & - & - & - & - & $-10.74(\mathrm{~d}, 16.1)^{e}$ & - \\
\hline
\end{tabular}




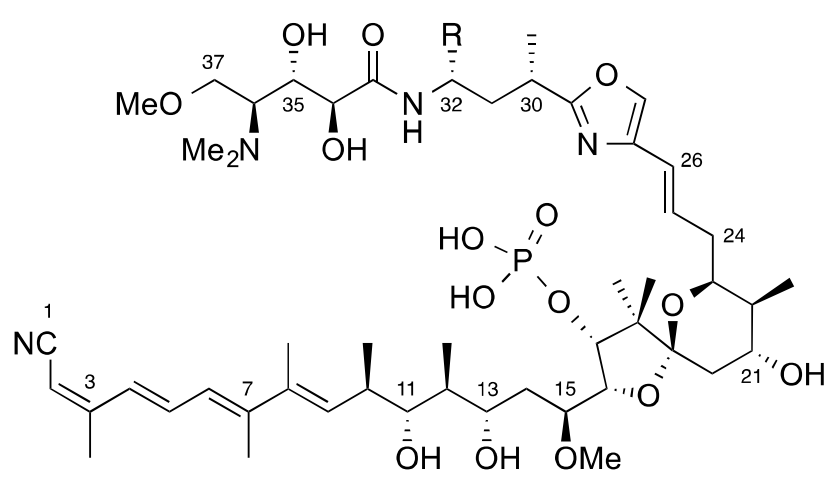

Calyculin $\mathrm{A}(\mathrm{R}=\mathrm{H}, \mathbf{1})$

Calyculin $C(R=M e, 2)$

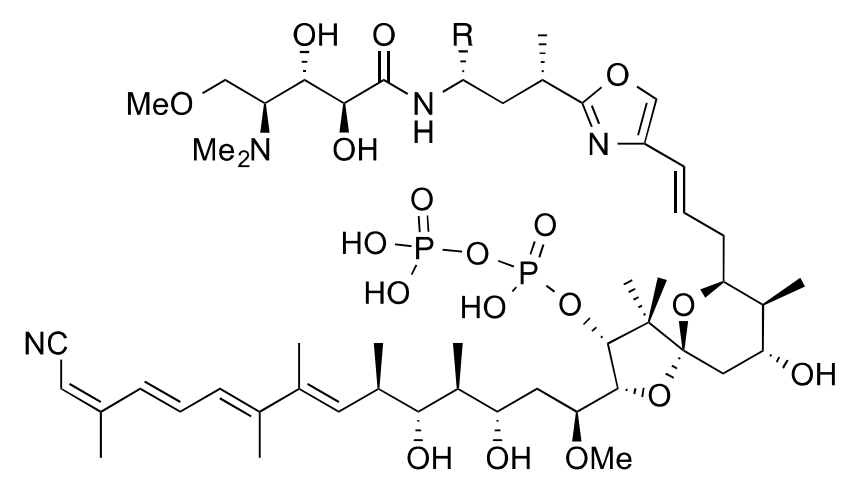

Phosphocalyculin A $(R=H, 3)$

Phosphocalyculin $\mathrm{C}(\mathrm{R}=\mathrm{Me}, 4)$

Figure 1. Structures of calyculin derivatives. 


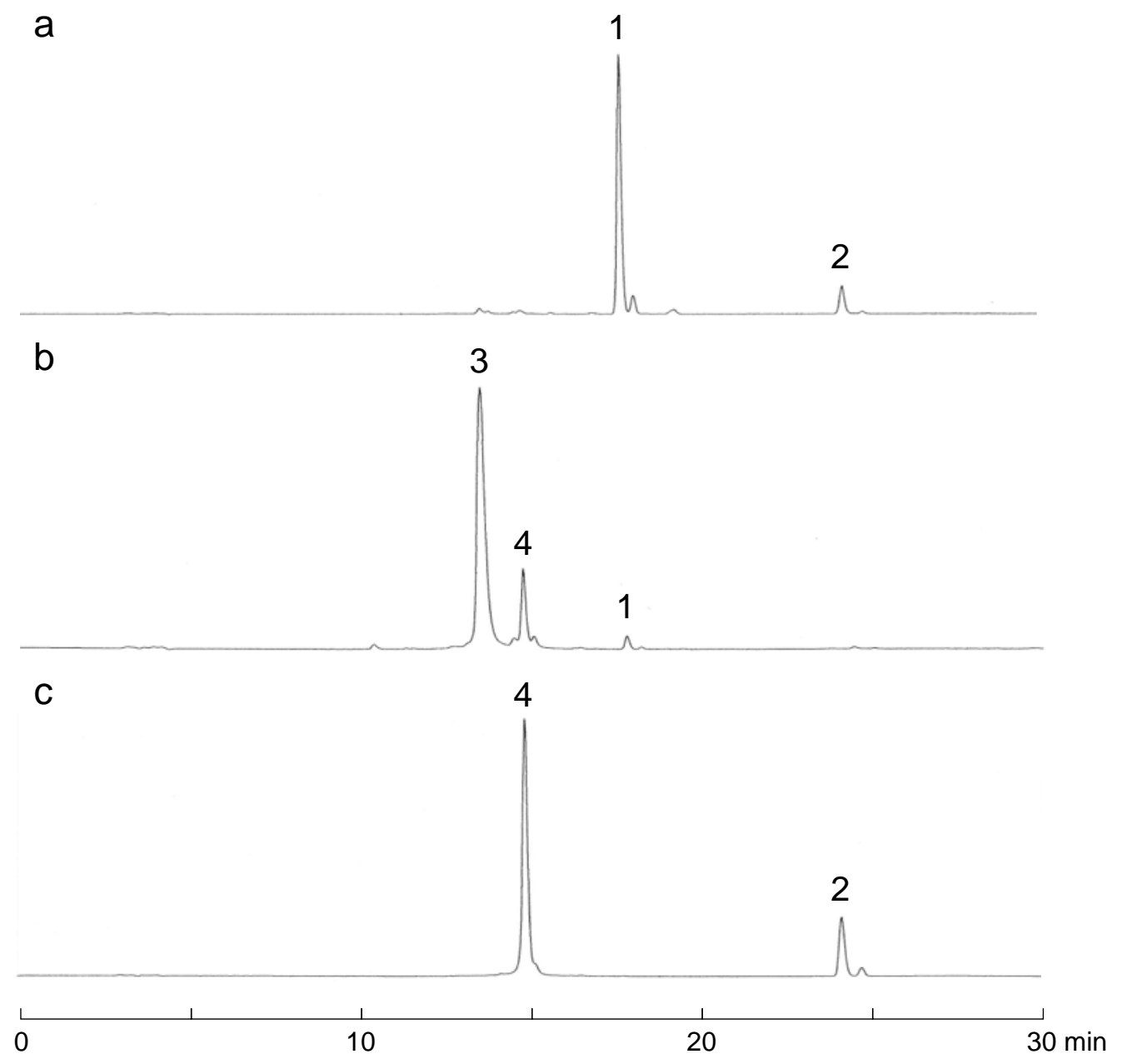

Figure 2. HPLC profiles of methanol extracts from D. calyx and the CalQ-catalyzed reaction. (a) The methanol extract of the frozen sponges, (b) the methanol extract of the sponges lyophilized after flash-freezing in liquid nitrogen, and (c) the product of the CalQ-catalyzed reaction with 2 as the substrate. All chromatograms were detected by UV at $340 \mathrm{~nm}$. 


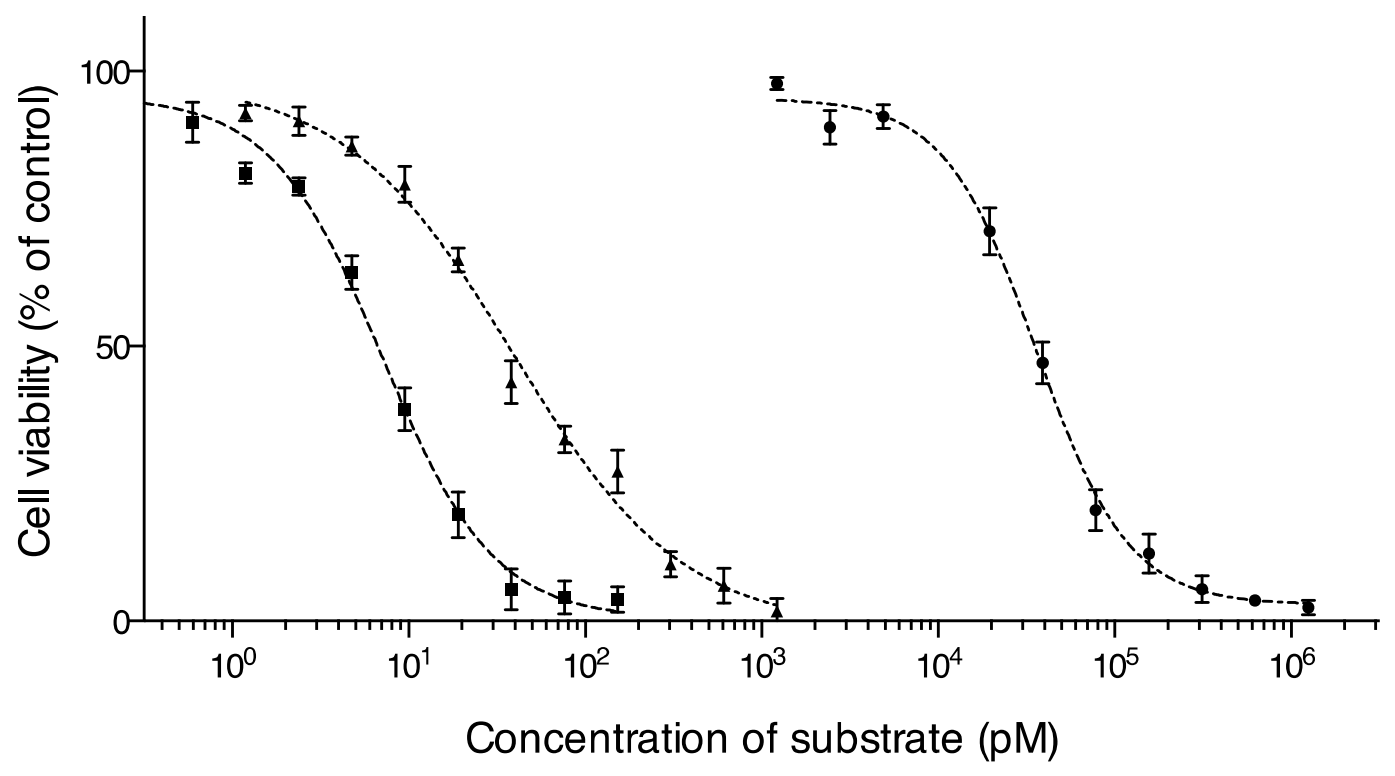

Figure 3. Cytotoxicity against $\mathbf{P 3 8 8}$ cells.

Cell viability curve of $\mathrm{P} 388$ cells treated with phosphocalyculin $\mathrm{C}$ (circles, $\mathrm{IC}_{50}: 36 \mathrm{nM}$ ), calyculin $\mathrm{C}$ (squares, $\mathrm{IC}_{50}: 7 \mathrm{pM}$ ), and phosphocalyculin $\mathrm{C}$ incubated with the $D$. calyx cell-free extract (triangles, $\left.\mathrm{IC}_{50}: 39 \mathrm{pM}\right)$. Data shown are mean values \pm S.D. $(\mathrm{n}=4)$. 